\section{SM Journal of Urology}

\author{
Article Information \\ Received date: Oct 05, 2016 \\ Accepted date: Oct 07, 2016 \\ Published date: Oct 10, 2016
}

\section{*Corresponding author}

Neelima Dhingra, Department of Pharmaceutical Chemistry, University Institute of Pharmaceutical Sciences, Punjab University, Chandigarh-160014, India, Tel: +91-9876573312;

Email: neelimad08@gmail.com

Distributed under Creative Commons CC-BY 4.0

Article DOI 10.36876/smju.1019

\section{Finasteride in Prostate Cancer: A Hope or Hype}

\author{
Neelima Dhingra* \\ Department of Pharmaceutical Chemistry, University Institute of Pharmaceutical Sciences; Punjab \\ University, India
}

The guest editors of this special issue and research scientist all across the globe, working on urology problems and prostate cancer have been asked by the editorial board of the SM journal of urology to collect and publish articles on prostate cancer so as to document the latest developments in this field. The intricacies and challenges of tackling this disease continue to puzzle and fascinate us. This particular issue on prostate cancer includes original research works, reviews, commentaries as well as debate and opinion pieces.

Increasing incidences of prostate cancer demanded an effective strategy to check its unabated occurrences. Chemoprevention seemed to be holding such potential. Finasteride an inhibitors of $5 \alpha$-reductase $(5 \alpha-R)$, by inhibiting conversion of testosterone to dihydrotestosterone (a potent androgen), with low toxicity, is an attractive chemopreventive agent and the drug has been proven successful by the U.S. Food and Drug Administration (FDA) in 1992, in treating Benign Prostatic Hyperplasia (BPH) [1]. Its effect is associated with a reduction in PSA levels [2], which prompted further studies to determine whether this agent may mitigate the risk of prostate cancer in men aging 55 and older [3].

Decades of experience has taught us to resist the temptation of blowing trumpets at early impressive results considering them as breakthroughs and the jubilations was short lived by the observations of Prostate Cancer Preventive Trial (PCPT), a large randomized clinical trial testing Finasteride in early-stage prostate cancer and raised the questions about how well that lesson has been learnt. PCPT suggested paradoxically, that the $5 \alpha$-RI Finasteride decreases the risk of lowgrade prostate cancer, but at the same time increased the risk of high-grade cancer. The observation by the Thompson IM Jr a prostate cancer specialist at University of Texas Health Science Center at, San Antonio, in NEJM journal described the results as simply stunning considering the fact that a higher percentage of men taking Finasteride had aggressive tumors than men in the placebo group $6.4 \%$ versus $5.1 \%[4]$.

PCPT findings were further confirmed by a recent follow-up study (up to 18 years) and it demonstrated not only high-grade prostate cancer was more common in the Finasteride group compared with placebo, but Finasteride had no significant effect on overall survival [5]. This indicates that Finasteride treatment may mask early detection of low-grade tumors by reducing PSA levels, thereby reducing the incidence of 'over diagnosis' (early detection of cancers that may never cause harm in a person's lifetime).

Owing to increased risks of high grade prostate cancer complications, it provided a timely reminder that medicines are not without their risks. We have known about the potential harm associated with medicines for centuries. Ideally we should protect patients from harm, but practically whenever a new molecule is approved, it remains impossible to know about its longterm repercussions. And gathering every bit of information prior to approval could delay access to potentially useful therapies for patients who have no other options-a delay, many cancer patients cannot afford. In 2011, the FDA announced and released 'the revised $5 \alpha$-RI with their new safety information and about the increased risk of being diagnosed with a more serious form of prostate cancer (high-grade prostate cancer)'.

The widespread acceptance of statins and aspirin for cardiovascular disease prevention proves that patients are willing to take a drug to prevent a disease which they may never get. In the case of Finasteride (5-ARI), patients are willing to take Finasteride with its preferential prevention of low-grade prostate, despite at the cost of small risk of reversible sexual side effects. The non-biased counseling of patients on the risks and benefits associated with the $5 \alpha$-reductase inhibitors and their potential for chemoprevention of prostate cancer is built on the clinical impact of the PCPT trials [6].

No physician or regulatory body is comfortable treating healthy men with a drug that has even the slightest risk of inducing a potentially lethal cancer. Until more is learned, physicians are unfortunately left with a difficult option of explaining the complicated risks and benefits of $5 \alpha$ - 
RI, and while it was never approved for chemopreventive use before, there has always been the hope that they would do so. And these divergent findings about the risk of high grade prostate cancer are preventing FDA approval and wider adoption as chemopreventive agent for prostate cancer.

As controversy still surrounds the probability of the $5 \alpha-\mathrm{RI}$ in impairing prostate cancer, additional studies are warranted to determine a safe, efficacious, cost-effective chemopreventive agent to be given to young men, for preventing the disease in the ageing population. Additional clinical trials need to be carried out to clarify the effect of exposure to the medicine in 'real life' situations and to define new indications. Better mechanisms are needed for reporting adverse drug reactions and we need to raise professional and public awareness about potential safety concerns.

Urology would definitely not exist as a separate specialization without PC and PC-specific treatments. The editors and guest editors of this special issue of the SM journal urology are therefore grateful to have the opportunity to draw more attention to the most frequent urological disease. We thank all authors who have accepted our invitation and shared their professional insights with us. We wish our readers a joyful and interesting time reading.

\section{Reference}

1. Tian G, Mook RA, Moss ML, Frye SV. Mechanism of time-dependent inhibition of 5 alpha-reductase by delta 1-4-azasteroids: Toward perfection of rates of time-dependent inhibition by using ligand-binding energies. Biochemistry. 1995; 34: 13453-13459.

2. Guess HA, Gormley GJ, Stoner E, Oesterlinq JE. The effect of finasteride on prostate specific antigen: review of available data. J Urol. 1996; 155: 3-9.

3. Thompson IM Jr, Goodman PJ, Tangen CM, Parnes HL, Minasian LM, Godley PA, et al. Long-term survival of participants in the prostate cancer prevention trial. N Engl J Med. 2013; 369: 603-610.

4. Channing JP, Thomas JS. Finasteride and Prostate Cancer: A Commentary, The Oncologist 2012; 17: 888-890.

5. McCarthy M. Finasteride for prostate cancer prevention has no effect on survival, study finds. Bio Med Journal 2013; 347: 5203.

6. Lacy JM, Kyprianou N. A tale of two trials: The impact of $5 \alpha$-reductase inhibition on prostate cancer, Oncology Lett. 2014; 8: 1391-1396. 